\title{
Recurrent vulval abscess caused by Actinomyces species: A case report
}

\author{
DM Casather
}

\section{Introduction}

Escherichia coli are the most commonly isolated organism causing vulval abscesses. In addition, anaerobes, micro-aerophilic bacteria, Bacteroides species and Prevotella species are frequently identified in vulval abscesses. Actinomycosis is a rare disease in humans and it was almost exclusively found in women with intra uterine contraceptive devices in place. Here we report a case of recurrent vulval abscesses due to the Actinomyces species.

\section{Case Report}

A 17-year-old schoolgirl presented to the gynaecology unit with vulval pain and swelling for two days. On examination, there was a large abscess on the left labia majora. In addition, there were ipsilateral, extremely tender, grossly enlarged inguinal lymph nodes. She has had vulval abscesses in same site on two previous occasions within the previous year. On both those occasions she had positive cultures for Actinomyces species; however, she did not receive a complete course of antibiotics. On this presentation, the patient had undergone incision and drainage and during the surgery, a copious amount of purulent exudate was evacuated. The patient had an uneventful recovery, and she was treated with oral cefuroxime and metronidazole for seven days. One week later pus culture was positive for Actinomyces species, and she was treated with another course of oral erythromycin $500 \mathrm{mg} 6$ hourly for 14 days.

There are a few cases of Actinomyces causing vulval abscess reported in the literature. Our case illustrates recurrent vulval abscesses in a young girl caused by Actinomyces species. Usually, Actinomyces vulval lesions appear as single or multiple indurated masses. Diagnosis of Actinomyces species is difficult, as even one dose of antimicrobials prior to bacterial culture can obscure test results. Actinomyces infections usually need long term treatment with penicillin. For patients with penicillin allergy, erythromycin, tetracycline, or clindamycin can be administered

\section{Conclusion}

In conclusion, Actinomyces species may rarely produce vulvar lesions and it should be kept mind when treating a patient who has recurrent vulval lesions with poor response to treatment.

Keywords: Actinomyces, Vulval abscess

National Hospital, Kandy, Sri Lanka, Sri Lanka

Address for correspondence: dilanmalinda7@yahoo.com. +0447448057371 Email: dilanmalinda7@yahoo.com (iD https://orcid.org/0000 000330580483 